\title{
Does Voiding Cystourethrogram Exclude Posterior Urethral Valves in Late Presenting Cases?
}

\author{
Mehmet Ali Özen ${ }^{1}$ Mehmet Taşdemir ${ }^{2}$ Gökhan Gündoğdu ${ }^{1} \quad$ Ilmay Bilge $^{2}$ Cenk Büyükünal ${ }^{3}$ \\ Egemen Eroğlu ${ }^{1}$ \\ ${ }^{1}$ Department of Pediatric Surgery, Koç University School of Medicine, \\ Istanbul, Turkey \\ 2 Department of Pediatric Nephrology, Koç University School of \\ Medicine, Istanbul, Turkey \\ ${ }^{3}$ Department of Pediatric Urology, Cerrahpaşa School of Medicine, \\ Istanbul University, İstanbul, Turkey \\ Eur J Pediatr Surg 2019;29:85-89. \\ Address for correspondence Egemen Eroğlu, MD, Department of \\ Pediatric Surgery, Koç University School of Medicine, Topkapı, \\ Maltepe 34010, Istanbul, Turkey \\ (e-mail: egemene@amerikanhastanesi.org).
}

\begin{abstract}
Keywords

- late-presenting

- posterior urethral valves

- diagnosis

- voiding cystourethrography

- cystoscopy

Introduction Delayed presentation of posterior urethral valves (PUVs) is a rare condition. Presentation and diagnosis of the patients with late PUVs are challenging. Voiding cystourethrogram (VCUG) is mainly practiced. In this study, we aimed to evaluate the children with late-presented PUVs, and the reliability of VCUG in this group.

Materials and Methods Between January 2003 and December 2017 records of patients who were diagnosed with late-presented PUVs were analyzed. Delayed presentation of PUV was defined as patients who were diagnosed and treated after infancy. Cases were examined in terms of age at diagnosis, presenting symptoms, urinalysis, urinary ultrasound, urodynamic studies, VCUG, and dimercaptosuccinic acid scintigraphy findings. Postoperative follow-up conditions were also assessed.

Results Seventeen boys were diagnosed with late-presented PUVs (mean age was 7.35 years). The most common symptoms at presentation were frequency (58.8\%), day and nighttime incontinence (47\%), and febrile urinary infection (41\%). PUV was noted by VCUG in 10 patients alone. The classical sign of dilated posterior urethra was detected in 9 patients. The 10th patient had posterior urethral irregularity. Urethra could not be evaluated due to unsuccessful voiding in one patient. Six patients had normally appearing urethra on VCUG. Reflux was detected in nine $(52.9 \%)$ patients. Conclusion Late-presented PUVs may be missed on VCUG. Whether a PUV might be present is crucial in boys with a history of recurrent urinary infection, persistent reflux, and repetitive daytime incontinence. Based on our results, we conclude that cystoscopic examination should be preferred for those cases to diagnose PUVs regardless of VCUG results.
\end{abstract}

\section{Introduction}

Posterior urethral valves (PUVs) are the most common form of congenital lower urinary tract (LUT) obstruction in boys, with an incidence of 1 case per 3,000 to 25,000 live births. ${ }^{1-4}$ When Young and McKay ${ }^{5}$ described PUV, they reported that

received

May 2, 2018

accepted after revision

August 13, 2018

published online

September 28, 2018

the mean age at presentation was 8.6 years; however, today, PUV diagnosis is mostly done prenatally or at birth because of advances in sonography. ${ }^{6,7}$ Although the delayed presentation of PUV is rare, it may still occur in $10 \%$ of cases. ${ }^{8,9}$ Presentation and diagnosis of the patients with late PUVs are nonspecific or challenging because of the variability and

(c) 2019 Georg Thieme Verlag KG Stuttgart - New York
DOI https://doi.org/ 10.1055/s-0038-1672146. ISSN 0939-7248. 
subtlety of the symptoms in older boys. ${ }^{10}$ Both early and delayed presentation of PUVs are important causes of chronic renal failure, so it is important for the physician to be informed about the existence of PUV.

Voiding cystourethrogram (VCUG) is commonly used in pediatric practice by pediatricians, pediatric nephrologists, pediatric surgeons, and urologists, and is mainly practiced in patients with recurrent urinary tract infections (UTIs), vesicoureteral reflux (VUR), and PUV. Worldwide, pediatricians are in agreement that if an urethra appears to be normal on VCUG, no urethral obstruction is present. ${ }^{11}$ In this study, we aimed to evaluate the children with delayed presentation of PUVs and the reliability of VCUG in this group.

\section{Materials and Methods}

An approval statement was obtained from the Koç University Hospital local ethics committee for the study. Between January 2003 and December 2017, field records of patients who are diagnosed with delayed presentation of PUVs were analyzed retrospectively at Koç University Hospital and VKF American Hospital. Delayed presentation of PUV was defined as patients who were diagnosed and treated after infancy. Patients, who were diagnosed prenatally or who were presented and diagnosed postnatally during the early infancy period before 1 year of age, were not included in the study.

Cases were examined in terms of age at diagnosis, presenting symptoms including UTI, the history of voiding habits, and the presence of incontinence. We used voiding dysfunction symptom score ${ }^{12}$ as a standard evaluation for LUT dysfunction. Urinalysis, serum creatinine level, urinary system ultrasound assessment, urodynamic studies (including video urodynamic study [VUDS], uroflowmetry-electromyography [UF/EMG] test), VCUG, and dimercaptosuccinic acid (DMSA) scintigraphy findings were evaluated. According to our pediatric nephro-urology council policy, a radiologist, interested in pediatric radiology and particularly pediatric urinary system radiology assessments, evaluated the VCUGs as a routine data. Estimated glomerular filtration rate (eGFR) was calculated by Schwartz formula as follows: $k \times$ length $(\mathrm{cm}) /$ serum creatinine level, $k=0.413) .{ }^{13}$ Operation reports and valve types were recorded. Postoperative follow-up conditions were also assessed.

\section{Results}

Seventeen boys were diagnosed with late-presented PUVs (mean age was 7.35 years, range: 2-12) from January 2003 to December 2017. The most common symptoms at presentation were LUT symptoms (LUTS), including urinary frequency ( $n: 10,58.8 \%)$, day and nighttime incontinence ( $n: 8,47 \%)$, history of burning or dysuria ( $n$ : $6,35.2 \%$ ), urgency/urge incontinence ( $n: 4,23.5 \%)$, abdominal straining ( $n: 2,11.7 \%)$, and hesitancy ( $n: 2,11.7 \%$ ). Monosymptomatic nocturnal enuresis was not noted as the only one symptom. The other presenting symptoms were febrile UTI ( $n$ : 7, 41\%), asymptomatic bacteriuria ( $n: 4,23.5 \%)$, proteinuria ( $n: 3,17.6 \%)$, and hematuria ( $n: 1,5.8 \%$ ) (-Table 1 ).
Table 1 Symptoms at presentation (17 patients)

\begin{tabular}{|l|l|}
\hline Urinary frequency & $10(58.8 \%)$ \\
\hline Day and nighttime incontinence & $8(47 \%)$ \\
\hline Febrile urinary tract infection & $7(41 \%)$ \\
\hline Burning or dysuria & $6(35.2 \%)$ \\
\hline Asymptomatic bacteriuria & $4(23.5 \%)$ \\
\hline Urgency/urge incontinence & $4(23.5 \%)$ \\
\hline Proteinuria & $3(17.6 \%)$ \\
\hline Hesitancy & $2(11.7 \%)$ \\
\hline Straining & $2(11.7 \%)$ \\
\hline Hematuria & $1(5.8 \%)$ \\
\hline
\end{tabular}

The serum creatinine levels, at diagnosis, were greater than the age-reference range in two patients (11.7\%). Their eGFR levels were 77.1 and $51.5 \mathrm{~mL} / \mathrm{min} / 1.73 \mathrm{~m}^{2}$.

Urinary ultrasound examination was performed in all patients and revealed hydronephrosis ( $n$ : 5 ), hydroureteronephrosis ( $n$ : 4), bladder wall thickening ( $n: 4)$, bladder diverticula ( $n: 2)$, postvoid residual (PVR) urine ( $n: 4)$, and normal findings ( $n: 4)$.

VCUG was obtained in all patients. On VCUG, PUV was reported in 10 (58.8\%) of the patients. The classical sign of dilated posterior urethra was detected in 9 patients. Posterior urethral irregularity was noticed in one patient. Urethra could not be evaluated due to unsuccessful voiding in one patient. Six patients had normally appearing urethra on VCUG (-Fig. 1). Only one patient had difficulty, during catheterization at the time of VCUG. Reflux was detected in 9 (52.9\%) patients of whom 6 had dilated posterior urethra and $14(41.1 \%)$ of 34 renal units; the most reflux was grade 3 (-Table 2). Other VCUG findings that we found in our patients were bladder trabeculation in 3 patients and bladder diverticula in 2 patients of which 1 had dilated posterior urethra.

Urodynamic studies were available in 10 patients and revealed detrusor overactivity ( $n$ : 5 ), significant PVR volume ( $n$ : 3), decreased cystometric bladder capacity ( $n: 4)$, and increased bladder capacity ( $n$ : 1$)$. Voiding pattern was documented as staccato ( $n$ : 4$)$ or interrupted ( $n$ : 4$)$. Two patients could not void during the procedure. UF/EMG test was performed in 6 patients. Plateau voiding pattern was detected in 2 patients without significant EMG activity. In addition, staccato ( $n: 2)$ and interrupted pattern ( $n: 2)$ without significant EMG activities were documented. Although the main purpose of this study was to evaluate the reliability of the VCUG on patients with PUVs presenting late, we have found that our invasive urodynamics studies were much more than noninvasive studies. In recent years, we prefer more noninvasive UF/EMG in our cases.

DMSA scintigraphy was performed in 11 (64.7\%) patients with VUR and/or with a history of febrile UTI, and 7 (63.6\%) patients had renal scarring.

Although there were normal urethral findings on VCUG, cystoscopy was performed in six children due to a history of recurrent UTI, persistent unilateral or bilateral reflux, and repetitive LUTS such as daytime and urgency/urge incontinence. 


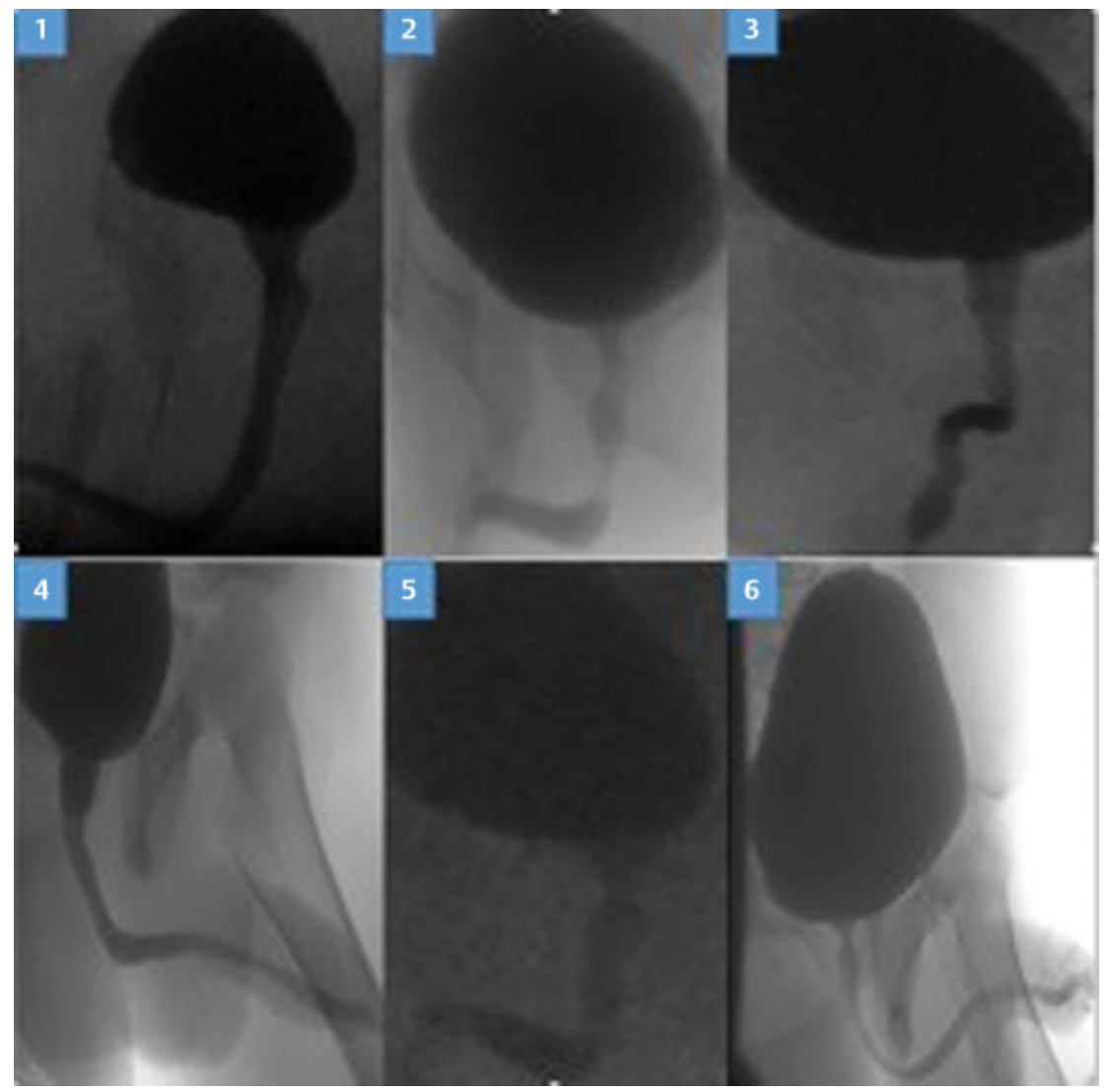

Fig. 1 Images of 6 patients with normal urethral appearance on voiding cystourethrogram but detected posterior urethral valve in cystoscopy.

Owing to our hospital policy, two pediatric surgeons and/ or urologist performed the operations. Type 1 PUV was the most common valve form in cystoscopic examination $(n: 13$, $76.4 \%)$, followed by type 3 PUV ( $n$ : $4,23.5 \%)$. The cold knife and electrocautery ablation were used to remove the valves.

The mean follow-up period was 52.8 months (range: 2-170). All patients were followed up with the pediatric nephrology

Table 2 The results of VCUG (17 patients)

\begin{tabular}{|l|l|}
\hline PUV noted & $10(58.8 \%)$ \\
\hline Dilated posterior urethra & $9(52.9 \%)$ \\
\hline Posterior urethral irregularity & $1(5.8 \%)$ \\
\hline Normal urethral appearance & $6(35.2 \%)$ \\
\hline Trabeculation & $3(17.6 \%)$ \\
\hline Bladder diverticula & $2(11.7 \%)$ \\
\hline VUR (17 patients) & $9(52.9 \%)$ \\
\hline VUR (34 renal units) & $14(41.1 \%)$ \\
\hline Grade I 1 & \\
\hline Grade II 3 & \\
\hline Grade III 5 & \\
\hline Grade IV 2 & \\
\hline Grade V 3 & \\
\hline
\end{tabular}

Abbreviations: PUV, posterior urethral valve; VCUG, voiding cystourethrogram; VUR, vesicoureteral reflux. department. During the follow-up period, LUTS have been improved in the 11 of 13 patients, and urinary frequency improved in 8 of 10 patients. Urinary incontinence improved in 10 of 12 patients; two patients have been receiving medical treatment (anticholinergics) and biofeedback urotherapy due to daytime and nighttime incontinence. PVR urine reduced in 2 of 4 patients with timed and double voiding urotherapy. In the other two patients, PVR reduced by using $\alpha-1$ adrenergic blocker. After valve ablation, 9 of 14 refluxing renal units resolved in following VUDS which were performed at 6 to 12 months. Remaining five refluxing ureters were managed as follows: a cystoscopic injection was performed in three units and ureteroneocystostomy was necessary for two units (due to high-grade persistent reflux and UTI). Diverticula were located on the left and right lateral walls of the bladder in two patients, not involved within the ureteral orifices. One of them underwent bilateral ureteroneocystostmy due to high-grade persistent VUR, and UTI diverticulectomy was also performed. Another patient was followed with good clinical course and diverticula did not require additional intervention.

There were no patients with new developing renal failure during the follow-up, except the two patients who have had renal insufficiency at diagnosis.

\section{Discussion}

Currently, PUVs are detected most frequently by prenatal ultrasonography. Delayed presentation of PUV is a rare 
condition and the number of studies related with this topic is lacking in the literature. In these studies, late presentation is generally defined after 1 year of age. ${ }^{14-16}$ We also defined delayed presentation of PUV as the patients presented beyond infancy (after 1 year of age). The factors causing delayed presentation and diagnosis are still vague. The presenting symptoms of delayed presentation of PUVs are age-dependent, nonspecific, and were thought to differ from early diagnosed PUV. ${ }^{17,18}$ Bomalaski et al reported that the most common presenting symptoms were daytime wetting (60\%), UTI (40\%), and urinary straining/pain (10\%). ${ }^{4,19}$ Zornoza et al reported daytime incontinence as the most common symptom in their study involving a total of 8 patients. ${ }^{16}$ In another study, Schober et al reported the presenting symptoms as nocturnal enuresis in $67 \%$, urinary frequency in $60 \%$, UTI in $17 \%$, and febrile UTI only in $3 \%$ patients. ${ }^{20}$ In our study, the mean age at presentation of late PUV was 7.35 years (range: 2-12) and symptoms were nonspecific and variable in these older boys group. The main three presenting symptoms were urinary frequency (58.8\%), incontinence (both day and nighttime incontinence and urge incontinence in $70.5 \%$ ), and UTI (febrile UTI was detected in $41.8 \%$ of patients and asymptomatic bacteriuria was in $23.5 \%$ ). Differently, from previously published series, no patient presented with monosymptomatic nocturnal enuresis alone.

Until recently, it was thought that early diagnosis had a worse prognosis. The delayed presentation has been described as a favorable prognostic sign suggesting a lesser degree of obstruction in children with PUVs. ${ }^{21-24}$ Currently, there is a controversy about the prognosis of children with PUVs presenting late. Reinberg et al showed that a poor prognosis was associated with neonatal diagnosis in 5 of 8 cases progressing to renal failure. ${ }^{25}$ In contrast to these studies, Tejani et al noted a worse outcome in children in whom the diagnosis was delayed beyond the age of 2 years, with 6 of 7 cases progressing to renal failure. ${ }^{26}$ In a retrospective review, Bomalaski et al reported the renal insufficiency in 35\% and end-stage renal disease in $10 \%$ in patients aged between 5 and 35 years with late PUVs. ${ }^{19}$ A recent systematic review and meta-analysis found that $22 \%$ of patients with PUV go on to develop chronic kidney failure. ${ }^{4}$ Although, late presentation of PUV may lead to chronic renal failure, in our study the patients had a better long-term prognosis than the ones reported in the literature. Decreased renal function was documented only in two patients (11.7\%) at diagnosis (eGFR levels were 77.1 and $51.5 \mathrm{~mL} / \mathrm{min} / 1.73 \mathrm{~m}^{2}$ ). There were no patients with new developing renal failure during follow-up.

Type 1 PUV was the most common valve form in cystoscopic examinations ( $n$ : $13,76.4 \%$ ), followed by type 3 PUV ( $n$ : $4,23.5 \%$ ). The high rate of type 3 valve in patients with late-presented PUV was the remarkable feature of this study. Although we did not make such a comparison between valve types in terms of prognosis in this study, we observed that the prognosis of type 3 valves was better than type 1 . The better prognosis may be related with higher rate of type 3 PUV in children with late-presented PUV in this study.

In this study, the mean follow-up period was 52.8 months. Currently, there is no standard approach to the management of the late presented PUVs. Some clinicians advocate for routine repeat VCUG after initial incision to remove any concern for residual valve tissue. ${ }^{14,27}$ We do not perform VCUG in the postoperative follow-up period. We routinely perform a control cystoscopy, 3 to 4 weeks after the ablation. However, the role of VUDS in the management of the PUVs is controversial, after 6 to 12 months of valve ablation, we perform VUDS for patients who have PUVs with VUR. ${ }^{4,14,27,28}$ Presence of VUR in PUV patients, is a potential indicator for eventual renal function loss. ${ }^{27} \mathrm{~A}$ review of the literature reports the presence of VUR in 22 to $67 \%$ of boys with PUV prior to valve ablation. ${ }^{28}$ Reflux was detected in 9 (52.9\%) patients and 14 of 34 renal units with PUV before valve ablation in this study. After valve ablation, 9 of 14 refluxing renal units resolved in the following VUDS which were performed at 6 to 12 months. The prominent clinical presentation was LUTS in our study group. Improvement in these symptoms of the patients was noted after valve ablation in 11 of 13 patients. Overall, long-term follow-up outcomes after valve ablation in delayed presentation of PUVs are variable.

A true reference standard for the diagnosis of urethral obstruction of boys does not exist. Cohen et al used transperineal ultrasonography to investigate PUVs in 10 boys and diagnosed valves on the basis of urethral dilation. ${ }^{29}$ Bosio and Manzoni also concluded that PUVs could be effectively visualized by transperineal ultrasonography with echo contrast. ${ }^{30}$ However, there are opinions that perineal ultrasonography is not suitable for detecting PUVs. ${ }^{31}$

Worldwide, a normally appearing posterior urethra in VCUG is widely accepted to exclude PUV in children, which subsequently makes cystoscopic examination unnecessary. ${ }^{11}$ The diagnosis of PUVs is suggested on VCUG. ${ }^{19,28}$ It is considered that VCUG remains the gold standard test for evaluating the urethra. ${ }^{16}$ But in contrast to these suggestions, the main outcome of this study is that there was a poor correlation between the presence of PUVs and the VCUG findings in the delayed presentation group. VCUG was performed in all patients. PUV was detected only in 10 (58.8\%) of 17 patients. Dilation of the posterior urethra on VCUG, which is the typical finding of PUVs, was found only in 9 children. One patient had posterior urethral irregularity on VCUG. The appearance of the urethra was normal in 6 patients on VCUG and PUV was not noted in these cases. Other VCUG findings that we found in our patients were VUR in 9 patients of which 6 had dilated posterior urethra, trabeculated bladder in 3 patients, and bladder diverticula in 2 patients of whom 1 had dilated posterior urethra. The question of whether a PUV might be present is crucial in boys with a history of recurrent UTI, persistent uni- or bilateral reflux, and repetitive LUTS such as daytime and urgency/urge incontinence. It is important to be informed about the existence of PUV because its presence has major implications for further therapy and prognosis. The detection rate of PUVs on VCUG was $58.8 \%$ in this study. Therefore, we thought that VCUG may not be a reliable instrument for excluding the possibility of PUVs in the late period, as present in our study. Diagnosis of delayed presentation of PUV patients is still controversial. Based on our results, we conclude that direct inspection with cystoscopy may be the reference standard for those cases to diagnose or exclude PUVs in older boys. It is an outpatient procedure, does 
not contain radiation, and it allows to identify the presence of PUVs as well as to treat it at the same time. We do not propose cystoscopy to all boys with LUTS since VCUG is not reliable. This raises the important question of when to perform cystoscopy in boys. We thought that persistent uni- or bilateral reflux in older boys, recurrent UTI, and daytime/urge incontinence that is resistant to the therapy might be considered an indication to perform cystoscopy.

\section{Conclusion}

Presentation and diagnosis of the patients with late PUVs are challenging because of the variability and subtlety of the symptoms in older boys. VCUG is the radiological method to assess PUVs, but may lead to misdiagnosis in patients with delayed presentation. According to the results of this study, it appears that VCUG is not an excellent method to detect late PUVs. A cystoscopic examination should be preferred if a boy has repetitive daytime and urge incontinence, recurrent UTI, and persistent unilateral or bilateral reflux regardless of VCUG results.

Prognosis of delayed presentation of PUVs is variable, and reports on the long-term follow-up after valve ablation of late-presenting PUVs are insufficient.

\section{Conflict of Interest}

None.

\section{References}

1 Yohannes P, Hanna M. Current trends in the management of posterior urethral valves in the pediatric population. Urology 2002;60(06):947-953

2 Nasir AA, Ameh EA, Abdur-Rahman LO, Adeniran JO, Abraham MK. Posterior urethral valve. World J Pediatr 2011;7(03):205-216

3 Casale AJ. Early ureteral surgery for posterior urethral valves. Urol Clin North Am 1990;17(02):361-372

4 Keays MA, Mcalpine K, Welk B. All grown up: a transitional care perspective on the patient with posterior urethral valves. Can Urol Assoc J 2018;12(04, Suppl 1):S10-S14

5 Young HH, McKay RW. Congenital valvular obstruction of the prostatic urethra. Surg Gynecol Obstet 1929;48:509-542

6 Tsingoglou S, Dickson JA. Lower urinary obstruction in infancy. A review of lesions and symptoms in 165 cases. Arch Dis Child 1972;47(252):215-217

7 Kaefer M, Barnewolt C, RetikAB, Peters CA. The sonographic diagnosis of infravesical obstruction in children: evaluation of bladder wall thickness indexed to bladder filling. J Urol 1997;157(03):989-991

8 Young HH, Frontz WA, Baldwin JC. Congenital obstruction of the posterior urethra. J Urol, 3: 289-365, 1919. J Urol 2002;167(01): 265-267

9 Pieretti RV. The mild end of the clinical spectrum of posterior urethral valves. J Pediatr Surg 1993;28(05):701-704

10 Dutkiewicz S. Posterior urethral valves in an adult male. A case report. Int Urol Nephrol 1994;26(05):555-558

11 de Jong TP, Radmayr C, Dik P, Chrzan R, Klijn AJ, de Kort L; Pediatric Urology Club Meeting, Stans, Austria, January 2007. Posterior urethral valves: search for a diagnostic reference standard. Urology 2008;72(05):1022-1025

12 Akbal C, Genc Y, Burgu B, Ozden E, Tekgul S. Dysfunctional voiding and incontinence scoring system: quantitative evaluation of incontinence symptoms in pediatric population. J Urol 2005; 173(03):969-973

13 Schwartz GJ, Brion LP, Spitzer A. The use of plasma creatinine concentration for estimating glomerular filtration rate in infants, children, and adolescents. Pediatr Clin North Am 1987;34(03): 571-590

14 Kibar Y, Ashley RA, Roth CC, Frimberger D, Kropp BP. Timing of posterior urethral valve diagnosis and its impact on clinical outcome. J Pediatr Urol 2011;7(05):538-542

15 Ansari MS, Singh P, Mandhani A, et al. Delayed presentation in posterior urethral valve: long-term implications and outcome. Urology 2008;71(02):230-234

16 Zornoza M, Angulo JM, Parente A, Simal S, Burgos L, Ortiz R. Late diagnosis of posterior urethral valves. Actas Urol Esp 2015;39 (10):646-650

17 Mahadik P, Vaddi SP, Godala CM, Sambar V, Kulkarni S, Gundala R. Posterior urethral valve: delayed presentation in adolescence. Int Neurourol J 2012;16(03):149-152

18 Jesus CM, Trindade Filho JC, Goldberg J. Late presentation of posterior urethral valve: two case reports. Sao Paulo Med J 2008;126(02): 126-127

19 Bomalaski MD, Anema JG, Coplen DE, Koo HP, Rozanski T, Bloom DA. Delayed presentation of posterior urethral valves: a not so benign condition. J Urol 1999;162(06):2130-2132

20 Schober JM, Dulabon LM, Woodhouse CR. Outcome of valve ablation in late-presenting posterior urethral valves. BJU Int 2004;94(04):616-619

21 Parkhouse HF, Barratt TM, Dillon MJ, et al. Long-term outcome of boys with posterior urethral valves. Br J Urol 1988;62(01): 59-62

22 Hendren WH. Posterior urethral valves in boys. A broad clinical spectrum. J Urol 1971;106(02):298-307

23 Duckett JW. Editorial comment to: the mild end of the clinical spectrum of posterior urethral valves. J Pediatr Surg 1993; 28:704-705

24 Jalbani IK, Biyabani SR. Late presentation of posterior urethral valves. J Coll Physicians Surg Pak 2014;24(02, Suppl 2):S155-S156

25 Reinberg Y, de Castano I, Gonzalez R. Prognosis for patients with prenatally diagnosed posterior urethral valves. J Urol 1992;148 (01):125-126

26 Tejani A, Butt K, Glassberg K, Price A, Gurumurthy K. Predictors of eventual end stage renal disease in children with posterior urethral valves. J Urol 1986;136(04):857-860

27 Long CJ, Bowen DK. Predicting and modifying risk for development of renal failure in boys with posterior urethral valves. Curr Urol Rep 2018;19(07):55

28 Concodora CW, Reddy PP, VanderBrink BA. The role of video urodynamics in the management of the valve bladder. Curr Urol Rep 2017;18(03):24

29 Cohen HL, Susman M, Haller JO, Glassberg KI, Shapiro MA, Zinn DL. Posterior urethral valve: transperineal US for imaging and diagnosis in male infants. Radiology 1994;192(01):261-264

30 Bosio M, Manzoni GA. Detection of posterior urethral valves with voiding cystourethrosonography with echo contrast. J Urol 2002; 168(4 Pt 2):1711-1715

31 de Kort LM, Uiterwaal CS, Beek EJ, Jan Nievelstein RA, Klijn AJ, de Jong TP. Reliability of voiding cystourethrography to detect urethral obstruction in boys. Urology 2004;63(05):967-971 